\title{
CUGC for Duchenne muscular dystrophy (DMD)
}

\author{
David J. Coote ${ }^{1,2} \cdot$ Mark R. Davis $^{3} \cdot$ Macarena Cabrera $^{4} \cdot$ Merrilee Needham ${ }^{5,6} \cdot$ Nigel G. Laing $^{1,2,3} \cdot$ \\ Kristen J. Nowak ${ }^{2,7,8}$
}

Received: 17 January 2017 / Revised: 8 August 2017 / Accepted: 9 September 2017 / Published online: 12 January 2018

(c) European Society of Human Genetics 2018

\section{Disease characteristics}

\subsection{Name of the disease (synonyms)}

Duchenne muscular dystrophy (DMD; Meryon's disease).

\subsection{OMIM\# of the disease}

310200.

\subsection{Name of the analysed genes or DNA/ chromosome segments}

Dystrophin (DMD).

Kristen J. Nowak

kristen.nowak@health.wa.gov.au

1 Centre for Medical Research, Faculty of Health and Medical Sciences, The University of Western Australia, Perth, WA 6009, Australia

2 Harry Perkins Institute of Medical Research, QQ Block, QEII Medical Centre, Nedlands, WA, Nedlands 6009, Australia

3 Neurogenetics Laboratory, Department of Diagnostic Genomics, PP Block, QEII Medical Centre, Nedlands, WA 6009, Australia

4 Department of Neurology and Instituto de Biomedicina de Sevilla, Hospital Universitario Virgen del Rocío, 41013 Seville, Spain

5 Perron Institute for Neurological and Translational Science, RR Block, QEII Medical Centre, Nedlands, WA 6009, Australia

6 Fiona Stanley Hospital, 11 Robin Warren Drive, Murdoch, WA 6150, Australia

7 School of Biomedical Sciences, Faculty of Health and Medical Sciences, The University of Western Australia, Perth, WA 6009, Australia

8 Department of Health, Public and Aboriginal Health Division, Office of Population Health Genomics, East Perth, WA 6004, Australia

\subsection{OMIM\# of the Gene(s)}

*300377

Review of the analytical and clinical diagnostic, validity as well as of the clinical utility of DNA-based testing for mutations in the $D M D$ gene in

predictive and prenatal settings and for

risk assessment in relatives.

\subsection{Mutational Spectrum}

Variants in the dystrophin gene (DMD) can cause Duchenne muscular dystrophy (DMD) or Becker muscular dystrophy (BMD). As the DMD gene is located on the $\mathrm{X}$ chromosome, these diseases almost exclusively affect males. The phenotype is most often dependent on whether the diseasecausing variant results in an absence of protein (DMD), or an abnormal protein size (BMD). However, certain diseasecausing variants located in integral domains can result in the translation of protein that is evidently non-functional based on clinical phenotype [1-3]. Atypical phenotypes include isolated quadriceps myopathy; [4] asymptomatic hyperCKemia; [5] myalgia, cramps and rhabdomyolysis; [6] dilated cardiomyopathy; [7] isolated cognitive impairment; [8] and symptomatic female carriers [9].

Boys with DMD usually present with motor delay at the age of 3 to 5 years. Examination at this age typically shows hypertrophy of calves and proximal skeletal muscle weakness indicated by lumbar hyperlordosis and the use of Gower's manoeuvre arising from the floor. Motor skills, although delayed, usually progress during the first years of life followed by a plateau phase of variable length after 
which motor deterioration starts. Myocardial involvement develops at a variable age. Respiratory and orthopaedic complications can arise, and non-progressive cognitive impairment may also be present in some boys. The wide use of steroid treatment has changed the natural history of the disease, with a delay in the onset of motor deterioration from 5 to 7 years, loss of ambulation from 9 to 12-14 years and prolonging survival to the $4^{\text {th }}$ decade of life. Ventilation aids have also contributed to prolonged life expectancy [1012].

BMD is more clinically heterogeneous and less severe in the majority of cases. Half of affected males present by age 10 with a limb-girdle pattern of skeletal muscle weakness, often with calf hypertrophy. Other affected individuals may present with comparatively mild weakness associated with adult-onset, or (very rarely) asymptomatic elevated serum creatine kinase (CK) levels. Cardiomyopathy is common, while cognition is usually spared. Loss of ambulation can occur as early as the teens, and typically in the third or fourth decade of life. Mildly affected patients never lose ambulation [13].

DMD is most frequently caused by 'out-of-frame' deletions or duplications in which the open reading frame is disturbed, leading to premature translation termination [14]. BMD typically has in-frame deletions or duplications leading to an internally deleted or expanded, yet partially functional protein [14]. Deletion of one or more exons exists in approximately $65 \%$ of DMD patients [15-17], with a minor hotspot existing between exons 3-19 and a major hotspot between exons 45-52 [18, 19] (exons numbered as per NG_012232.1 and the Leiden Open Variation Database). Exonic duplications account for a further 6-10\% of disease-causing variants $[13,20]$.

Small variants perturbing the reading frame (nonsense, indel, splice variants) and missense variants can also cause DMD [13]. Missense variants can result in DMD rather than BMD if the variant affects the actin binding domain $[2,21]$ or the $\beta$-dystroglycan binding domain of dystrophin [1]. There are other rare cases of in-frame variants resulting in DMD rather than BMD, e.g. large in-frame deletions at the $\mathrm{N}$-terminus and extending into the rod domain of dystrophin can disrupt actin binding and result in a DMD phenotype [22]. Regularly updated public $D M D$ variant databases can be located at the Leiden Open Variation Databases (http:// www.dmd.n1/nmdb2/home.php?select_db=DMD) and ClinVar (https://www.ncbi.nlm.nih.gov/clinvar/).

\subsection{Analytical methods}

Parents of boys with DMD typically seek medical attention for their child between the ages of 3 and 5 years [13], although prenatal [23], and newborn [24] testing is available to families with confirmed X-linked carrier status. The first postnatal diagnostic test is generally serum CK level, which is always elevated (usually between 50 and 100 times normal levels) [25]. Detailed mutational analysis is then generally performed. The average age of diagnosis is 4.5-4.11 years [26].

Exon copy number can be assessed through a number of techniques [27-29], with multiplex ligation-dependent probe amplification (MLPA) [30] and array comparative genomic hybridization (array $\mathrm{CGH}$ ) being the favoured methods. These techniques are usually the first genetic analyses to be performed for a patient with suspected DMD. Then for cases where an exon copy number variant is not identified, or in positive cases where the patient is to be considered for exon-skipping therapy, sequencing of the entire coding region from genomic DNA should be performed [31]. Next generation sequencing (NGS) strategies facilitate this process and can include sub-exomic targeted gene panels or whole exome sequencing [32-34].

It is envisaged that whilst NGS approaches will become increasingly utilised to determine a genetic diagnosis, the possibility of atypical variants must continue to be considered for selected cases. For example, if no likely diseasecausing variants have been identified in $D M D$ (or another skeletal muscle gene if a gene panel has been used or whole exome sequencing performed) then cDNA analysis of DMD can occur. This typically requires RNA to be extracted from a skeletal muscle biopsy; however, a streamlined approach to transcript analysis can be via non-invasive skin biopsies to detect deletions/duplications of two or more base pairs and variants affecting splicing [35].

Indeed altered (absent) dystrophin expression in a skeletal muscle specimen remains the gold standard supporting evidence for DMD. However if a previously characterised DMD-causing variant is identified in an individual, and symptomology is consistent with a DMD diagnosis, further diagnostic testing of a skeletal muscle biopsy is not always required [10]. This is often also the case for variants categorised as class 5 or class 4 , and can prevent a skeletal muscle biopsy being taken.

Supportive evidence from a skeletal muscle biopsy (e.g. dystrophin immunostaining, cDNA analysis) is still necessary when a variant of unknown significance (class 3 ) in the $D M D$ gene is identified, or in the case of a negative genetic test following clinical suspicion of DMD [10]. Additionally point variants occurring deep within intronic regions of $D M D$ also usually require detection through skeletal muscle biopsy, followed by sequencing of cDNA derived from muscle mRNA, and then directed genomic DNA analysis based on the cDNA sequencing results [13]. 


\subsection{Analytical validation}

For suspected DMD patients with elevated CK levels (including newborns), and/or those patients with negative dystrophin immunostaining on muscle biopsies, genetic analysis is required to provide a definitive molecular diagnosis [24]. If a single exon is duplicated or deleted, this finding can be verified using an independent method to exclude technical artefacts and rare sequence variants affecting proper probe binding [36]. Mutational analysis should not be relied upon solely to offer a DMD or BMD classification. Instead, the clinical picture should be considered as a whole, taking into account family history, age of onset, phenotypic presentation and, if available, dystrophin expression within a muscle biopsy [13].

\subsection{Estimated frequency of the disease}

The estimated frequency of DMD worldwide is 1 in 3600 , to 1 in 6000 live male births [37, 38]. DMD predominantly affects males due to the DMD gene being present on the $\mathrm{X}$ chromosome; however, rare female cases can exist due to skewing of $\mathrm{X}$ chromosome inactivation [39].

\subsection{Diagnostic setting}

\begin{tabular}{lcc}
\hline & Yes. & No. \\
A. (Differential) diagnosis & $\mathrm{X}$ & \\
B. Predictive testing & $\mathrm{X}$ & \\
C. Risk assessment in relatives & $\mathrm{X}$ \\
D. Prenatal & $\mathrm{X}$ & \\
\hline
\end{tabular}

Comment:

Diseases that could be mistaken for DMD include several congenital muscular dystrophies and limb girdle muscular dystrophies, Pompe disease (OMIM 232300), dermatomytosis and BMD (OMIM 300376) [40].

Female carrier status can be determined using the same technique/s as with the index patient (usually MLPA, followed by DNA sequencing) [36]. It is desirable to characterise the variant in the index patient or obligate carrier before testing at-risk relatives.

One third of all $D M D$ variants arise de novo in female carriers or in index patients [13]. Therefore family members of these individuals (excluding offspring) have no elevated risk of developing DMD [41]. A variant from an index case that does not appear in the respective mother's somatic cells may in fact be present as a mosaic in the mother's oocytes [36]. Offspring arising from mothers with a germline variant would have an elevated risk of developing
DMD or being carriers depending on the degree of germinal mosaicism.

\section{Test characteristics}

$\begin{array}{ll}\begin{array}{l}\text { Genotype or A: True positives } \\ \text { disease }\end{array} & \begin{array}{l}\text { Calse } \\ \text { negative }\end{array} \\ \text { Present Absent B: False positives } & \begin{array}{l}\text { D: True } \\ \text { negative }\end{array}\end{array}$

Test

$\begin{array}{llll}\text { Positive A } & \text { B } & \text { Sensitivity: } & \mathrm{A} /(\mathrm{A}+\mathrm{C}) \\ & & \begin{array}{l}\text { Specificity: } \\ \text { Negative C }\end{array} & \mathrm{D} /(\mathrm{D}+\mathrm{B}) \\ & & \begin{array}{l}\text { Positive predic- } \\ \text { tive value: }\end{array} & \mathrm{A} /(\mathrm{A}+\mathrm{B}) \\ & \begin{array}{l}\text { Negative predic- } \\ \text { tive value: }\end{array} & \mathrm{D} /(\mathrm{C}+\mathrm{D}) \\ & & \end{array}$

\subsection{Analytical sensitivity (proportion of positive tests if the genotype is present)}

Close to $100 \%$. The analytical sensitivity of MLPA alone is $\sim 71 \%$ and $\sim 97 \%$ when combined with sequencing of the coding regions and splice sites following negative MLPA results [36]. Studies using single platform mutational analysis have reported sensitivities between 92 and 99\% [32, 34, 42]. Deep intronic variants can be characterised through directed sequencing based on results from sequencing cDNA derived from skeletal muscle mRNA [43].

\subsection{Analytical specificity (proportion of negative tests if the genotype is not present)}

Close to $100 \%$.

\subsection{Clinical sensitivity (proportion of positive tests if the disease is present)}

If a correct clinical diagnosis has been made then the disease-causing $D M D$ variant is identified in close to $100 \%$ of cases. However, $~ 25 \%$ of patients referred for molecular diagnosis due to a suspicion of DMD are found to be negative for disease-causing $D M D$ variants, and some of these when tested by a neuromuscular disease gene panel are determined to indeed have a disease-causing variant/s in a different gene (and therefore are given a revised diagnosis of another skeletal muscle disease). 


\subsection{Clinical specificity (proportion of negative tests if the disease is not present)}

Close to $100 \%$ for males. Female carrier status can be determined using the same techniques as for the index patient in the respective family [36].

\subsection{Positive clinical predictive value (life time risk to develop the disease if the test is positive)}

Assuming a positive test of an index case is an out of frame variant in $D M D$, there is a $91-96 \%$ of developing DMD (otherwise they may develop BMD, depending on the variant) [44]. If the variant is known to cause DMD in other family members, the chance of the index patient developing DMD is close to $100 \%$.

\subsection{Negative clinical predictive value (probability not to develop the disease if the test is negative)}

Index case in that family had been tested:

Close to $100 \%$. An individual could harbour an undetected $D M D$ variant other than that which has been characterised in his family. Case studies of families with two distinct variants exist $[45,46]$.

Index case in that family had not been tested:

Close to $100 \%$.

\section{Clinical utility}

\section{1 (Differential) Diagnostics: The tested person is clinically affected}

(To be answered if in 1.9 "A" was marked)

\subsubsection{Can a diagnosis be made other than through a genetic test?}

No. (continue with 3.1.4)

Yes.

Clinically
Physical examination as reviewed by Bushby and colleagues [10] can contribute to a diagnosis. Early symptoms include proximal weakness, calf enlargement, motor skill delay, and reduced timed functional testing results. However the usefulness of a clinical diagnosis is limited without a
Imaging

Endoscopy

Biochemistry Elevated serum CK levels.

Electrophysiology

Other (please describe):

family history and/or alternative diagnostic tests.

Histopathology. Dystrophin protein expression can be detected using dystrophin antibodies targeted against different regions of the dystrophin protein for either immunofluorescence or immunohistochemical staining of skeletal muscle biopsy sections. Absence of dystrophin protein and reduced/ truncated/dysfunctional dystrophin can be indicative of DMD or BMD. Utrophin is usually overexpressed in the sarcolemma [20]. Alternatively/additionally dystrophin antibodies can be utilised for western blot analysis of total protein extracted from skeletal muscle tissue. Western blot analysis can also detect a change in size of the dystrophin protein. Of note, DMD patients can have a small number of dystrophinpositive fibres within their skeletal muscle biopsies, which are known as 'revertant fibers' [47].

Whilst a classic clinical phenotype, elevated CK and/or the absence of dystrophin protein are highly indicative of a diagnosis of DMD, a definitive molecular diagnosis requires genetic testing.

\subsubsection{Describe the burden of alternative diagnostic methods to the patient}

Clinical and biochemical assessment can provide a preliminary diagnosis, although genetic testing is required to confirm the diagnosis, and to determine the disease-causing variant. Characterising the disease-causing variant in an index patient also simplifies predictive testing in family members. Histopathology can be useful in providing evidence towards understanding the disease mechanism, although skeletal muscle biopsy is invasive and considered traumatic by some families. Additionally, accurate genetic diagnosis is necessary for inclusion in variant-specific 
treatments currently in clinical trials (reviewed by Guiraud et al.) [48].

\subsubsection{How is the cost effectiveness of alternative diagnostic methods to be judged?}

As alternative diagnostic methods are not definitive and a molecular diagnosis remains the ultimate endpoint, they add extra cost to the diagnosis. However, for gene specific mutational analysis, alternative diagnostic methods can direct molecular analysis and might therefore be economically beneficial. Elevated CK levels, absent/diminished dystrophin staining and/or abnormal dystrophin size can also assist in analysis of NGS results (whole exome/genome sequencing or sub-exonic gene panels), possibly facilitating or expediting molecular diagnosis.

\subsubsection{Will disease management be influenced by the result of a genetic test?}

No.

Yes.

Therapy (please Prednisone and deflazacort (cortidescribe) costeroids) along with cardiac medication and ventilation aids are currently the standards of care for DMD [44, 49]. However, these therapies do not address the underlying genetic cause of DMD and their use is not reliant on variant identity [13]. Nevertheless, numerous novel therapies addressing fundamental molecular aspects of specific variant types are currently in clinical trials. For example, exon skipping by antisense oligonucleotides [50] and nonsense variant suppression [51] are both emerging therapies in clinical trials, with efficacy dependent on a specific genetic diagnosis. The Food and Drug Administration (USA) granted accelerated approval of the $D M D$ exon 51 skipping drug eteplirsen in September 2016, which is estimated to be applicable for $\sim 14 \%$ of DMD patients. Additionally, CRISPR-Cas9 approaches have shown promise in preclinical

\begin{tabular}{|c|c|}
\hline & $\begin{array}{l}\text { testing in patient-specific cell } \\
\text { transplantation therapy }[52,53] \\
\text { and dystrophin-deficient mouse } \\
\text { models [54-56]. }\end{array}$ \\
\hline $\begin{array}{l}\text { Prognosis (please } \\
\text { describe) }\end{array}$ & $\begin{array}{l}\text { Genetic testing is helpful in dif- } \\
\text { ferentiating between DMD and } \\
\text { BMD, the latter of which may } \\
\text { have a comparatively mild pheno- } \\
\text { type [13]. However, exceptions to } \\
\text { the 'reading-frame rule' [16, 44] } \\
\text { and certain variants being asso- } \\
\text { ciated with variable phenotypes } \\
\text { [14] make prognosis prediction } \\
\text { from genetic test results alone } \\
\text { unreliable. For example, out-of- } \\
\text { frame deletions of exons } 3-7 \text { can } \\
\text { cause BMD, DMD or an inter- } \\
\text { mediate phenotype, depending on } \\
\text { whether translation is reinitiated } \\
\text { downstream [57, 58]. }\end{array}$ \\
\hline $\begin{array}{l}\text { Management } \\
\text { (please describe) }\end{array}$ & $\begin{array}{l}\text { Disease management as elicited by } \\
\text { Bushby and colleagues [10, } 11] \text { is } \\
\text { not affected by a genetic diagnosis } \\
\text { beyond classification as DMD. } \\
\text { However, accurate genetic coun- } \\
\text { selling relies upon genetic testing. } \\
\text { Maternal DNA should be tested to } \\
\text { determine whether the mother is a } \\
\text { carrier of her child's variant. A } \\
\text { positive result here should result in } \\
\text { cascade testing of at risk female } \\
\text { relatives, whereas a negative result } \\
\text { does not rule out gonadal mosai- } \\
\text { cism, and genetic counselling must } \\
\text { be carried out with this in mind. }\end{array}$ \\
\hline
\end{tabular}

\subsection{Predictive setting: the tested person is clinically unaffected but carries an increased risk based on family history}

(To be answered if in 1.9 "B" was marked)

\subsubsection{Will the result of a genetic test influence lifestyle and prevention?}

If the test result is positive (please describe):

Patients are typically diagnosed due to phenotypic manifestation around 5 years of age [16], by which time motor skill decline may have begun. Current best practise recommends commencement of corticosteroid treatment 
during motor skill plateau, prior to decline [10]. Therefore, predictive testing, such as newborn screening could result in earlier corticosteroid treatment and thus improved prognosis [59, 60]. However, early childhood treatment regimens must be carefully controlled as corticosteroids have marked side effects including obesity, reduced bone density and early onset of glaucoma and cataracts [61]. Early diagnosis may also enhance the patient's chances of enrolment in a clinical trial for a novel therapy, which are thought to be most beneficial before substantial skeletal muscle mass is lost [62].

Positive predictive testing can also have a significant effect on the family. Parents can avoid the convoluted 'diagnostic odyssey', often taking up to 2 years post initial presentation [61]. Additionally, early diagnosis can prompt carrier testing in the mother (and subsequent familial cascade screening if the mother is positive for a diseasecausing $D M D$ variant) and allow parents the option of preimplantation or prenatal testing. Other familial lifestyle effects may include travel options pre-loss and post-loss of ambulation of the affected child and choice of family home (e.g. single story home as apposed to multi-story home; proximity to clinician and school, etc.).

If the test result is negative (please describe)

A negative test result for an at-risk male would most likely provide relief for his family in the following situations:

- prenatal testing of a fetus,

- if the infant was indicated as being at-risk via newborn screening using CK levels, or

- pre-symptomatic cascade screening of a boy.

A negative genetic test result for a potential carrier mother provides some reassurance in regards to future family planning, however, germline mosaicism needs to be considered.

3.2.2 Which options in view of lifestyle and prevention does a person at-risk have if no genetic test has been done (please describe)?

Alternative diagnostic tests are available which can be strongly indicative of DMD (see Section 3.1.1).

Parents should be critically aware of early signs of DMD, especially a delay in the onset of walking (defined as occurring at or after 16 months of age) [63]. Physical examination of at-risk children by an experienced physician can often resolve whether a disease phenotype is present.

If no molecular genetic diagnosis is available, then prevention of an affected child to an at-risk mother could occur by avoiding pregnancy altogether, choosing only female embryos for implantation via in vitro fertilisation techniques, or prenatal testing to determine the sex of the foetus. Only male foetuses would be at higher risk of having DMD than individuals in the general population.

With disease management improving and potential therapies being developed, it may become increasingly plausible for Duchenne patients to have children. Consequently, such couples may choose to select for male embryos (which would not inherit the $\mathrm{X}$ chromosome harbouring the $D M D$ disease-causing variant, and thereby not inherit DMD, from the father), as female offspring would be obligate carriers of the father's variant. There is an isolated report of a consanguineous couple conceiving a female Becker patient homozygous for a $D M D$ deletion [64]. While this scenario will remain extremely rare, it may factor into genetic counselling for males with DMD wishing to father children.

\subsection{Genetic risk assessment in family members of a diseased person}

(To be answered if in 1.9 "C" was marked)

\subsubsection{Does the result of a genetic test resolve the genetic situation in that family?}

The identification of a $D M D$ variant usually halts further mutational analysis. However, it is possible that a second unidentified variant exists that could contribute to pathogenicity in a family $[45,46]$.

\subsubsection{Can a genetic test in the index patient save genetic or other tests in family members?}

A conclusive genetic test in an index patient would characterise the almost-always singular DMD-causing variant in that family. As such, this may facilitate maternally related males who are manifesting a similar disease from avoiding other invasive tests. Maternally related females, including the mother of the index patient, should be tested to define their carrier status. Directed genetic testing can occur in presymptomatic, at-risk males.

\subsubsection{Does a positive genetic test result in the index patient enable a predictive test in a family member?}

Following a positive test result in a child, the mother should be tested for that variant to determine whether she is a carrier. However, negative peripheral blood DNA testing results do not rule out the possibility of maternal gonadal mosaicism; the associated risk of which can be as high as $10 \%[65,66]$ and should be discussed with a genetic counsellor. If the mother is a carrier, at risk relatives should be tested to determine carrier/disease status. 


\subsection{Prenatal diagnosis}

(To be answered if in 1.9 "D" was marked)

\subsubsection{Does a positive genetic test result in the index patient enable a prenatal diagnosis?}

Yes. Prenatal testing is usually only considered when the index patient's mother is a known/suspected carrier of DMD, and the disease-causing $D M D$ variant is known. However, germline mosaicism in the mother should be considered as a possibility. If there is no recorded family history and the variant has been identified in a potential mother, prenatal differential diagnosis between DMD and BMD can be unreliable due to exceptions to the reading frame rule.

\section{If applicable, further consequences of testing}

Please assume that the result of a genetic test has no immediate medical consequences. Is there any evidence that a genetic test is nevertheless useful for the patient or his/her relatives? (please describe)

An accurate genetic diagnosis (particularly one that is presymptomatic) can have a profound affect on the family of a boy with DMD. The long and convoluted clinical diagnostic process is essentially eliminated or significantly reduced, reducing the anxiety associated with misunderstanding a child's early symptoms [67]. Knowledge of the prognosis also allows the patient and their family time to prepare emotionally and practically for the onset of the disease. Finally, an early genetic diagnosis allows future reproductive choice and underpins informative genetic counselling.

Acknowledgements This work was supported by EuroGentest2 (Unit 2: "Genetic testing as part of health care"), a Coordination Action under FP7 (Grant Agreement Number 261469) and the European Society of Human Genetics. K.J.N. was funded by an Australian Research Council Future Fellowship FT100100734, N.G.L. by a National Health and Medical Research Council Principal Research Fellowship APP1002147 and M.C. by Instituto de Salud Carlos III (JR15/00042)

\section{Compliance with ethical standards}

Conflict of interest The authors declare that they have no conflict of interest.

\section{References}

1. Vulin A, Wein N, Strandjord DM, et al. The ZZ domain of dystrophin in DMD: making sense of missense mutations. Hum Mutat. 2014;35:257-64.

2. Prior TW, Papp AC, Snyder PJ, et al. A missense mutation in the dystrophin gene in a Duchenne muscular dystrophy patient. Nat Genet. 1993;4:357-60.

3. Becker K, Robb SA, Hatton Z, Yau SC, Abbs S, Roberts RG. Loss of a single amino acid from dystrophin resulting in Duchenne muscular dystrophy with retention of dystrophin protein. Hum Mutat. 2003;21:651.

4. Sunohara N, Arahata K, Hoffman EP, et al. Quadriceps myopathy: forme fruste of Becker muscular dystrophy. Ann Neurol. 1990;28: 634-9.

5. Melis MA, Cau M, Muntoni F, et al. Elevation of serum creatine kinase as the only manifestation of an intragenic deletion of the dystrophin gene in three unrelated families. Eur J Paediatr Neurol. 1998;2:255-61.

6. Veerapandiyan A, Shashi V, Jiang YH, Gallentine WB, Schoch K, Smith EC. Pseudometabolic presentation of dystrophinopathy due to a missense mutation. Muscle Nerve. 2010;42:975-9.

7. Nakamura A. X-linked dilated cardiomyopathy: a cardiospecific phenotype of dystrophinopathy. Pharmaceuticals. 2015;8:303-20.

8. North KN, Miller G, Iannaccone ST, et al. Cognitive dysfunction as the major presenting feature of Becker's muscular dystrophy. Neurology. 1996;46:461-5.

9. Giliberto F, Radic CP, Luce L, Ferreiro V, de Brasi C, Szijan I. Symptomatic female carriers of Duchenne muscular dystrophy (DMD): genetic and clinical characterization. J Neurol Sci. 2014;336:36-41.

10. Bushby K, Finkel R, Birnkrant DJ, et al. Diagnosis and management of Duchenne muscular dystrophy, part 1: diagnosis, and pharmacological and psychosocial management. Lancet Neurol. 2010;9:77-93.

11. Bushby K, Finkel R, Birnkrant DJ, et al. Diagnosis and management of Duchenne muscular dystrophy, part 2: implementation of multidisciplinary care. Lancet Neurol. 2010;9:177-89.

12. Eagle M, Bourke J, Bullock R, et al. Managing Duchenne muscular dystrophy--the additive effect of spinal surgery and home nocturnal ventilation in improving survival. Neuromuscul Disord. 2007; 17:470-5.

13. Wein N, Alfano L, Flanigan KM. Genetics and emerging treatments for Duchenne and Becker muscular dystrophy. Pediatr Clin N Am. 2015;62:723-42.

14. Monaco AP, Bertelson CJ, Liechti-Gallati S, Moser H, Kunkel LM. An explanation for the phenotypic differences between patients bearing partial deletions of the DMD locus. Genomics. 1988;2:90-5.

15. Flanigan KM, Dunn DM, von Niederhausern A, et al. Mutational spectrum of DMD mutations in dystrophinopathy patients: application of modern diagnostic techniques to a large cohort. Hum Mutat. 2009;30:1657-66.

16. Tuffery-Giraud S, Beroud C, Leturcq F, et al. Genotypephenotype analysis in 2,405 patients with a dystrophinopathy using the UMD-DMD database: a model of nationwide knowledgebase. Hum Mutat. 2009;30:934-45.

17. Dent KM, Dunn DM, von Niederhausern AC, et al. Improved molecular diagnosis of dystrophinopathies in an unselected clinical cohort. Am J Med Genet A. 2005;134A:295-8.

18. Koenig M, Hoffman EP, Bertelson CJ, Monaco AP, Feener C, Kunkel LM. Complete cloning of the Duchenne muscular dystrophy (DMD) cDNA and preliminary genomic organization of the DMD gene in normal and affected individuals. Cell. 1987;50:509-17. 
19. Den Dunnen JT, Grootscholten PM, Dauwerse JG, et al. Reconstruction of the $2.4 \mathrm{Mb}$ human DMD-gene by homologous YAC recombination. Hum Mol Genet. 1992;1:19-28.

20. Falzarano MS, Scotton C, Passarelli C, Ferlini A. Duchenne muscular dystrophy: from diagnosis to therapy. Molecules. 2015;20:18168-84.

21. Singh SM, Kongari N, Cabello-Villegas J, Mallela KMG. Missense mutations in dystrophin that trigger muscular dystrophy decrease protein stability and lead to cross- $\beta$ aggregates. Proc Natl Acad Sci USA. 2010;107:15069-74.

22. Matsumura K, Burghes AH, Mora M, et al. Immunohistochemical analysis of dystrophin-associated proteins in Becker/Duchenne muscular dystrophy with huge in-frame deletions in the NH2terminal and rod domains of dystrophin. J Clin Invest. 1994;93:99-105.

23. Yoo SK, Lim BC, Byeun J, et al. Noninvasive prenatal diagnosis of Duchenne muscular dystrophy: comprehensive genetic diagnosis in carrier, proband, and fetus. Clin Chem. 2015;61:829-37.

24. Mendell JR, Shilling C, Leslie ND, et al. Evidence-based path to newborn screening for Duchenne muscular dystrophy. Ann Neurol. 2012;71:304-13.

25. Zatz M, Rapaport D, Vainzof M, et al. Serum creatine-kinase (CK) and pyruvate-kinase (PK) activities in Duchenne (DMD) as compared with Becker (BMD) muscular dystrophy. J Neurol Sci. 1991;102:190-6.

26. van Ruiten HJ, Straub V, Bushby K, Guglieri M. Improving recognition of Duchenne muscular dystrophy: a retrospective case note review. Arch Dis Child. 2014;99:1074-7.

27. Prior TW, Bridgeman SJ. Experience and strategy for the molecular testing of Duchenne muscular dystrophy. J Mol Diagn. 2005;7:317-26.

28. Flanigan KM, von Niederhausern A, Dunn DM, Alder J, Mendell JR, Weiss RB. Rapid direct sequence analysis of the dystrophin gene. Am J Hum Genet. 2003;72:931-9.

29. del Gaudio D, Yang Y, Boggs BA, et al. Molecular diagnosis of Duchenne/Becker muscular dystrophy: enhanced detection of dystrophin gene rearrangements by oligonucleotide arraycomparative genomic hybridization. Hum Mutat. 2008;29:1100-7.

30. Janssen B, Hartmann C, Scholz V, Jauch A, Zschocke J. MLPA analysis for the detection of deletions, duplications and complex rearrangements in the dystrophin gene: potential and pitfalls. Neurogenetics. 2005;6:29-35.

31. Aartsma-Rus A, Ginjaar IB, Bushby K. The importance of genetic diagnosis for Duchenne muscular dystrophy. J Med Genet. 2016;53:145-51.

32. Lim BC, Lee S, Shin JY, et al. Genetic diagnosis of Duchenne and Becker muscular dystrophy using next-generation sequencing technology: comprehensive mutational search in a single platform. J Med Genet. 2011;48:731-6.

33. Wang Y, Yang Y, Liu J, et al. Whole dystrophin gene analysis by next-generation sequencing: a comprehensive genetic diagnosis of Duchenne and Becker muscular dystrophy. Mol Genet Genom. 2014;289:1013-21.

34. Wei X, Dai Y, Yu P, et al. Targeted next-generation sequencing as a comprehensive test for patients with and female carriers of DMD/BMD: a multi-population diagnostic study. Eur J Hum Genet. 2014;22:110-8.

35. Bovolenta M, Scotton C, Falzarano MS, Gualandi F, Ferlini A. Rapid, comprehensive analysis of the dystrophin transcript by a custom micro-fluidic exome array. Hum Mutat. 2012;33:572-81.

36. Grimm T, Kress W, Meng G, Müller CR. Risk assessment and genetic counseling in families with Duchenne muscular dystrophy. Acta Myol. 2012;31:179-83.

37. Emery AE. Population frequencies of inherited neuromuscular diseases--a world survey. Neuromuscul Disord. 1991;1:19-29.
38. Drousiotou A, Ioannou P, Georgiou T, et al. Neonatal screening for Duchenne muscular dystrophy: a novel semiquantitative application of the bioluminescence test for creatine kinase in a pilot national program in Cyprus. Genet Test. 1998;2:55-60.

39. Richards CS, Watkins SC, Hoffman EP, et al. Skewed X inactivation in a female MZ twin results in Duchenne muscular dystrophy. Am J Hum Genet. 1990;46:672-81.

40. Emery AEH, Muntoni F, Quinlivan RCM. Differential diagnosis. In: Emery AEH, Muntoni F, Quinlivan RCM, editors. Duchenne muscular dystrophy. 3rd ed. New York: Oxford University Press; 2003. p. 82-96.

41. Edwards JH. The population genetics of Duchenne: natural and artificial selection in Duchenne muscular dystrophy. J Med Genet. 1986;23:521-30.

42. Okubo M, Minami N, Goto K, et al. Genetic diagnosis of Duchenne/Becker muscular dystrophy using next-generation sequencing: validation analysis of DMD mutations. J Hum Genet. 2016;61:483-9.

43. Mah JK, Selby K, Campbell C, et al. A population-based study of dystrophin mutations in Canada. Can J Neurol Sci. 2011;38:465-74.

44. Bladen CL, Salgado D, Monges S, et al. The TREAT-NMD DMD Global Database: analysis of more than 7,000 Duchenne muscular dystrophy mutations. Hum Mutat. 2015;36:395-402.

45. Morandi L, Mora M, Tedeschi S, et al. DMD and BMD in the same family due to distinct mutations. Am J Med Genet. 1995;59:501-5.

46. Wilton SD, Johnsen RD, Pedretti JR, Laing NG. Two distinct mutations in a single dystrophin gene: identification of an altered splice-site as the primary Becker muscular dystrophy mutation. Am J Med Genet. 1993;46:563-9.

47. Thanh LT, Nguyen TM, Helliwell TR, Morris GE. Characterization of revertant muscle fibers in Duchenne muscular dystrophy, using exon-specific monoclonal antibodies against dystrophin. Am J Hum Genet. 1995;56:725-31.

48. Guiraud S, Chen H, Burns DT, Davies KE. Advances in genetic therapeutic strategies for Duchenne muscular dystrophy. Exp Physiol. 2015;100:1458-67.

49. Sejerson T, Bushby K. Standards of care for Duchenne muscular dystrophy: brief TREAT-NMD recommendations. Adv Exp Med Biol. 2009;652:13-21.

50. Touznik A, Lee JJ, Yokota T. New developments in exon skipping and splice modulation therapies for neuromuscular diseases. Expert Opin Biol Ther. 2014;14:809-19.

51. Bushby K, Finkel R, Wong B, et al. Ataluren treatment of patients with nonsense mutation dystrophinopathy. Muscle Nerve. 2014;50:477-87.

52. Li Hongmei L, Fujimoto N, Sasakawa N, et al. Precise correction of the dystrophin gene in Duchenne muscular dystrophy patient induced pluripotent stem cells by TALEN and CRISPR-Cas9. Stem Cell Rep. 2015;4:143-54.

53. Ousterout DG, Kabadi AM, Thakore PI, Majoros WH, Reddy TE, Gersbach CA. Multiplex CRISPR/Cas9-based genome editing for correction of dystrophin mutations that cause Duchenne muscular dystrophy. Nat Commun. 2015;6:6244.

54. Nelson CE, Hakim $\mathrm{CH}$, Ousterout DG, et al. In vivo genome editing improves muscle function in a mouse model of Duchenne muscular dystrophy. Science. 2016;351:403-7.

55. Tabebordbar M, Zhu K, Cheng JK, et al. In vivo gene editing in dystrophic mouse muscle and muscle stem cells. Science. 2016;351:407-11.

56. Xu L, Park KH, Zhao L, et al. CRISPR-mediated genome editing restores dystrophin expression and function in $\mathrm{mdx}$ mice. Mol Ther. 2016;24:564-9.

57. Malhotra SB, Hart KA, Klamut HJ, et al. Frame-shift deletions in patients with Duchenne and Becker muscular dystrophy. Science. 1988;242:755-9. 
58. Winnard AV, Mendell JR, Prior TW, Florence J, Burghes AHM. Frameshift deletions of exons 3-7 and revertant fibers in Duchenne muscular dystrophy: mechanisms of dystrophin production. Am J Hum Genet. 1995;56:158-66.

59. Merlini L, Cicognani A, Malaspina E, et al. Early prednisone treatment in Duchenne muscular dystrophy. Muscle Nerve. 2003;27:222-7.

60. Kinali M, Mercuri E, Main M, Muntoni F, Dubowitz V. An effective, low-dosage, intermittent schedule of prednisolone in the long-term treatment of early cases of Duchenne dystrophy. Neuromuscul Disord. 2002;12:169-74.

61. Ross LF. Screening for conditions that do not meet the Wilson and Jungner criteria: the case of Duchenne muscular dystrophy. Am J Med Genet A. 2006;140:914-22.

62. Laing NG, Davis MR, Bayley K, Fletcher S, Wilton SD. Molecular diagnosis of duchenne muscular dystrophy: past, present and future in relation to implementing therapies. Clin Biochem Rev. 2011;32:129-34.

63. Mirski KT, Crawford TO. Motor and cognitive delay in Duchenne muscular dystrophy: implication for early diagnosis. J Pediatr. 2014;165:1008-10.

64. Fujii K, Minami N, Hayashi Y, et al. Homozygous female Becker muscular dystrophy. Am J Med Genet A. 2009;149a:1052-5.

65. Barbujani G, Russo A, Danieli GA, Spiegler AW, Borkowska J, Petrusewicz IH. Segregation analysis of 1885 DMD families: significant departure from the expected proportion of sporadic cases. Hum Genet. 1990;84:522-6.

66. Grimm T, Muller B, Muller CR, Janka M. Theoretical considerations on germline mosaicism in Duchenne muscular dystrophy. J Med Genet. 1990;27:683-7.

67. Parsons E, Clarke A, Hood K, Lycett E, Bradley D. Newborn screening for Duchenne muscular dystrophy: a psychosocial study. Fetal Neonatal Ed. 2002;86:91-95. 